Journal Club

Editor's Note: These short, critical reviews of recent papers in the Journal, written exclusively by graduate students or postdoctoral fellows, are intended to summarize the important findings of the paper and provide additional insight and commentary. For more information on the format and purpose of the Journal Club, please see http://www.jneurosci.org/misc/ifa_features.shtml.

\title{
Temporally Selective Firing of Cortical and Thalamic Neurons during Sleep and Wakefulness
}

\author{
William M. Connelly and Adam C. Errington \\ Neuroscience Division, School of Biosciences, Cardiff University, Cardiff CF10 3AX, United Kingdom \\ Review of Ushimaru et al.
}

During non-REM sleep and anesthesia, the electroencephalogram is characterized by the occurrence of slow $(<1 \mathrm{~Hz})$ wave activity (SWA) that results from synchronous fluctuations in the membrane potential of populations of cortical neurons between quiescent, hyperpolarized "Down" states and depolarized "Up" states that are typically associated with action potential firing. The functional significance of this type of activity, known as the slow oscillation (SO), has until recently received little attention. However, it has been demonstrated that enhancing Up states during sleep improved consolidation of declarative memory (Marshall et al., 2006). Furthermore, patterns of action potential firing in cortical neurons during Up states reflect those during wakefulness (Euston et al., 2007), leading to the suggestion that Up states might represent fragments of wakefulness in which information processing occurs during non-REM sleep (Destexhe et al., 2007).

Since its discovery nearly 20 years ago, the SO has generally been thought to be generated almost entirely by the neocortex. This view is based upon three central pieces of evidence: (1) the neocortex can generate the SO in vivo after thalamic le-

\footnotetext{
Received March 9, 2012; revised April 5, 2012; accepted April 6, 2012.

This work was supported by Wellcome Trust Grant 091882 (awarded to V. Crunelli).

Correspondence should be addressed to Adam C. Errington, Neuroscience Division, School of Biosciences, Cardiff University, Museum Avenue, Cardiff CF10 3AX, UK. E-mail: erringtonac@cardiff.ac.uk.

DOI:10.1523/JNEUROSCI.1164-12.2012

Copyright $\odot 2012$ the authors $\quad 0270-6474 / 12 / 327415-03 \$ 15.00 / 0$
}

sions or destruction of cortical afferents, (2) the SO is lost in the thalamus after decortication, and (3) isolated neocortical brain slices can generate a form of SO, whereas it is generally reported that thalamocortical cells cannot. Nonetheless, there is increasing evidence that subcortical structures, in particular the thalamus, have a significant role in shaping SWA. First, bursting activity in thalamocortical neurons has been reported to occur slightly before neocortical Down-to-Up state transitions in cortico-thalamo-cortical slices (Rigas and Castro-Alamancos, 2007). Second, both thalamocortical and thalamic reticular neurons undergo SO in vitro when metabotropic glutamate receptors at corticothalamic synapses are repetitively or tonically activated (Hughes et al., 2002; Blethyn et al., 2006). Third, in vivo whisker stimulation during Down states is highly effective in triggering Up states in layer II/III barrel cortex neurons. These findings, among others, have lead to the hypothesis that, although neocortex can generate SO in isolation, the true manifestation of SWA relies on input from intrinsic thalamic oscillators, which in turn are driven by corticothalamic activity (Crunelli and Hughes, 2010).

Despite longstanding uncertainty in the mechanism of neocortical Up state generation and the role of the thalamus in SWA, an in-depth study into the relative timing of thalamic and neocortical cell firing during the SO has been lacking. In a recent article in The Journal of Neuroscience, Ushimaru et al. (2012) addressed this omission by performing simultaneous sin- gle unit and local field potential (LFP) recordings from identified neurons of several cortical [frontal layer $\mathrm{V}$ corticothalamic (CTh)] and thalamic [ventrolateral (VL), anterior/ventromedial (VA/VM), thalamic reticular nucleus (TRN)] regions during SWA in anesthetized rats (Fig. 1).

First, the authors demonstrated that SWA and spindle oscillations $(7-14 \mathrm{~Hz})$ in LFP recordings were highly synchronous across the deep layers of M2 cortex and that higher frequency spindle waves, which are thought to be generated in the TRN, were nested in Up states and absent during Down states. After simultaneous injection of retrograde and anterograde tracers into M2 cortex, the presence of labeled corticothalamic and thalamocortical fibers in the VL and VA/VM thalamic nuclei confirmed a reciprocal connection between these areas. Initially, Ushimaru et al. (2012) attempted to record from layer $2 / 3 \mathrm{~b}$ cortical neurons because these receive dense thalamic input (equivalent to laver IV in sensory cortical areas), but post hoc laminar identification based on juxtacellular labeling, immunohistochemistry, spike shape, and interspike intervals revealed that most neurons that fired during slow waves were, in fact, located in layer $V$. It appears from these data that layer $2 / 3 \mathrm{~b}$ neurons in M2 cortex fire infrequently during SWA. In contrast, cells in layer V, identified as CTh neurons via their antidromic response to thalamic stimulation, were found to be active during SWA.

The most important conclusions of Ushimaru et al. (2012) are derived from 
the detailed analysis of spike timing of CTh, VL, VA/VM, and TRN neurons relative to the Up and Down states of SWA. By comparing spike-frequency histograms for individual units with cortical LFP, the authors found that the peak firing rate of VA/VM and TRN neurons typically occurred shortly before Up state initiation (median time from Up state start: $0.2-0.25 \mathrm{~s}$ ), whereas VL and CTh neuron firing typically peaked significantly later (500-650 ms) (Ushimaru et al., 2012, their Fig. $6 B, C$ ). Furthermore, although firing rates in most neurons increased during Up states, only thalamic cells had periods of activity preceding the transition to Up states (Fig. 1). When Up state duration was normalized, the median firing times of VA/VM and TRN neurons were $37 \%$ and $34 \%$ into the Up state, respectively, whereas $\mathrm{CTh}$ and VL neurons fired $51 \%$ and $52 \%$ into the Up state, respectively. In fact, by analyzing the variance of firing in each region, it was found that specific neurons fire, on average, in a temporally specific manner, with VA/VM and TRN neurons tending to fire at the beginning of Up states whereas VL and CTh fire more uniformly throughout Up states (Ushimaru et al., 2012, their Fig. 7). During Down states, CTh cells only fired rarely whereas thalamic cells fired more regularly and usually near the end of the Down state, that is, immediately preceding Up state initiation.

Spindle rhythms are classically considered to be generated primarily by TRN, but are thought to be shaped and potentially evoked by cortical activity (Contreras and Steriade, 1996). The activity of thalamic and cortical units was probed using circular analysis with reference to the spindle wave recorded in the deep layers of M2 cortex. The negative trough of the spindle was defined as $0^{\circ}$. Although CTh cells fired, on average, near the trough of the spindle $\left(14^{\circ}\right)$, they were in fact only weakly modulated by spindle activity (angular concentration: 0.05$)$. VA/VM and VL cells fired later in the oscillation at $211^{\circ}$ and $229^{\circ}$, respectively, with VA/VM cells having a stronger angular concentration (0.13) than VL cells (0.09). TRN cells fired during the positive peak of the spindle wave $\left(188^{\circ}\right)$ and were the most strongly modulated (0.24). Because VA/VM cells target neocortical layer 1A while VL cells target layer $2 / 3 \mathrm{~b}$, spindle-frequencymodulated firing in VA/VM could hypothetically produce strong excitatory drive to apical dendritic tufts of layer $V$ neurons resulting in the occurrence of a current sink in layer $1 \mathrm{~A}$ and a current source in

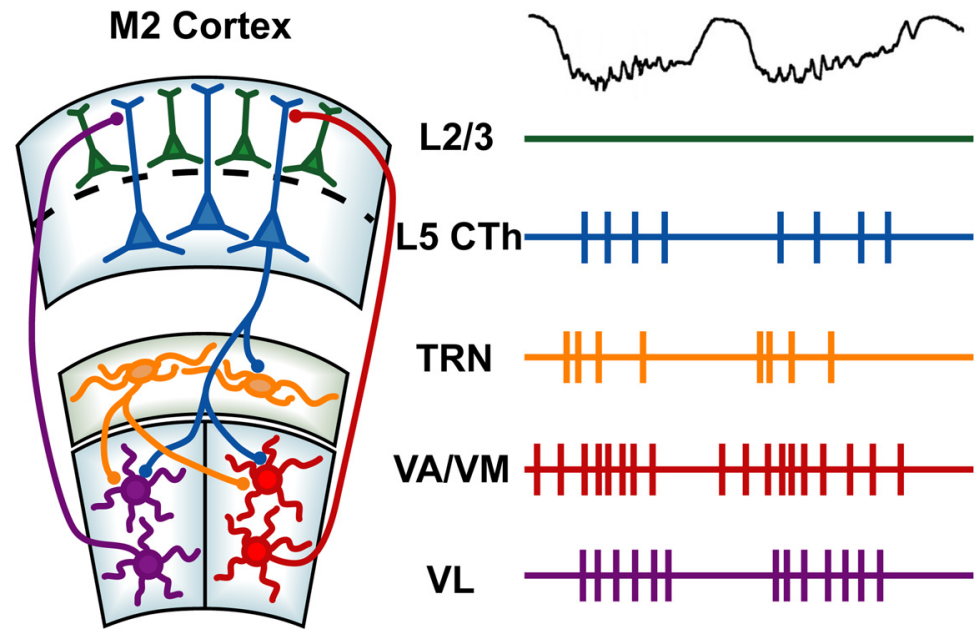

Figure 1. Thalamic neurons in VA/VM and TRN preferentially fire at the onset of cortical Up states. Conversely, CTh and VL neuron show little preference for different phases of the Up state and fire uniformly. VA/VM input might be an efficient mechanism to trigger cortical Up states by exciting cortical layer (L) 1 and apical dendritic tufts of layer 5 pyramidal neurons.

deeper layers with the polarity reversing in the middle layers. This is precisely what was observed in the data (Ushimaru et al., 2012, their Fig. 11). These data suggest that in M2 cortex, thalamic input to superficial cortical layers may be an efficient mechanism to generate Up states whereas the role of thalamic input into the classical recipient layer $2 / 3 \mathrm{~b}$ is minimal.

Finally, by analyzing spike timing during a period in which the effects of anesthesia were waning and the brain was entering a desynchronized state, Ushimaru et al. (2012) attempted to investigate the assertion that Up states represent neuronal activity equivalent to states of wakefulness. Under these conditions, it was found that layer V CTh neurons were significantly more active during UP states than during desynchronized activity. While these data present an interesting insight into the differences between desynchronized states and Up states and the authors suggest that Up states cannot be regarded as fragments of wakefulness as proposed by others (Destexhe et al., 2007), it is important to note that these findings are based on experiments in anesthetized animals. The desynchronized states in this study presumably reflect a lightly anesthetized state rather than natural wakefulness, because although slow waves and spindles had ceased, the authors report that the animals showed no signs of consciousness. It has been shown that properties of SO can vary markedly between anesthetized states and natural SWA (Chauvette et al., 2011) and therefore a full comparison of firing between true wakefulness and natural SWA may be better suited to fully address this question.
The thorough study by Ushimaru et al. (2012) presents further evidence that thalamic activity may play a pivotal role in shaping SWA characteristic of non-REM sleep. Because VA/VM activity often precedes initiation of the neocortical Up state, it is tempting to think that these thalamic cells may help to set the rhythm for cortical SOs. However, further experimental evidence would be required to reach this conclusion. Future studies, perhaps involving selective modulation or abolition of firing in VA/VM neurons using novel optogenetic techniques, may be able to fully answer this question. Much remains to be learned about the functional role of SO, but this work has undoubtedly contributed greatly to our understanding of mechanisms underpinning it.

\section{References}

Blethyn KL, Hughes SW, Tóth TI, Cope DW, Crunelli V (2006) Neuronal basis of the slow $(<1 \mathrm{~Hz})$ oscillation in neurons of the nucleus reticularis thalami in vitro. $\mathrm{J}$ Neurosci 26:2474-2486.

Chauvette S, Crochet S, Volgushev M, Timofeev I (2011) Properties of slow oscillation during slow-wave sleep and anesthesia in cats. J Neurosci 31:14998-15008.

Contreras D, Steriade M (1996) Spindle oscillation in cats: the role of corticothalamic feedback in a thalamically generated rhythm. J Physiol 490:159-179.

Crunelli V, Hughes SW (2010) The slow $(<1 \mathrm{~Hz})$ rhythm of non-REM sleep: a dialogue between three cardinal oscillators. Nat Neurosci 13:9-17.

Destexhe A, Hughes SW, Rudolph M, Crunelli V (2007) Are corticothalamic "up" states fragments of wakefulness? Trends Neurosci 30:334-342.

Euston DR, Tatsuno M, McNaughton BL (2007) Fast-forward playback of recent memory se- 
quences in prefrontal cortex during sleep. Science 318:1147-1150.

Hughes SW, Cope DW, Blethyn KL, Crunelli V (2002) Cellular mechanisms of the slow $(<1$ $\mathrm{Hz}$ ) oscillation in thalamocortical neurons in vitro. Neuron 33:947-958.
Marshall L, Helgadóttir H, Mölle M, Born J (2006) Boosting slow oscillations during sleep potentiates memory. Nature 444: 610-613.

Rigas P, Castro-Alamancos MA (2007) Thalamocortical Up states: differential effects of intrinsic and extrinsic cortical inputs on persistent activity. J Neurosci 27:4261-4272.

Ushimaru M, Ueta Y, Kawaguchi Y (2012) Differentiated participation of thalamocortical subnetworks in slow/spindle waves and desynchronization. J Neurosci 32:1730-1746. 